\title{
Measurement of the Width Difference of $B_{d}$ Mesons *
}

\author{
A.S. Dighe ${ }^{\mathrm{a}}$, T. Hurth ${ }^{\mathrm{b}}$, C.S. Kim ${ }^{\mathrm{c}}$ and T. Yoshikawa ${ }^{\mathrm{d}}$ \\ a Max-Planck-Institute for Physics, Föhringer Ring 6, D-80805 Munich, Germany \\ b CERN, Theory Division, CH-1211 Geneva 23, Switzerland \\ c Department of Physics and IPAP, Yonsei University, Seoul 120-749, Korea \\ d Department of Physics and Astronomy, University of North Carolina, \\ Chapel Hill, NC 27599-3255, USA
}

We estimate $\Delta \Gamma_{d} / \Gamma_{d}$, including $1 / m_{b}$ contributions and part of the next-to-leading order QCD corrections, and find it to be around $0.3 \%$. We show the methods to measure $\Delta \Gamma_{d} / \Gamma_{d}$ by using at least two different final states on the untagged $B_{d}$ decay. The nonzero width difference can also be used to identify new physics effects and to resolve a twofold discrete ambiguity in the $B_{d}-\bar{B}_{d}$ mixing phase. With the high statistics and accurate time resolution of the upcoming LHC experiment, the measurement of $\Delta \Gamma_{d}$ seems to be possible. This measurement would be important for an accurate measurement of $\sin 2 \phi_{1}$ at the LHC. We also derive an upper bound on the value of $\Delta \Gamma_{d} / \Gamma_{d}$ in the presence of new physics.

The neutral $B_{d}$ meson system has two mass eigenstates which have the mass difference and lifetime difference. The mass difference has been measured well but the lifetime difference has not been done because it is very tiny. Within the standard model (SM), the difference in the decay widths is CKM-suppressed with respect to that in the $B_{s}$ system. A rough estimate leads to $\frac{\Delta \Gamma_{d}}{\Gamma_{d}} \sim \frac{\Delta \Gamma_{s}}{\Gamma_{s}} \cdot \lambda^{2} \approx 0.5 \%$, where $\lambda=0.225$ is the sine of the Cabibbo angle, and we have taken $\Delta \Gamma_{s} / \Gamma_{s} \approx 15 \%$ [1] (see also [2,3]). Here $\Gamma_{d(s)}=$ $\left(\Gamma_{L}+\Gamma_{H}\right) / 2$ is the average decay width of the light and heavy $B_{d(s)}$ mesons $\left(B_{L}\right.$ and $B_{H}$ respectively). We denote these decay widths by $\Gamma_{L}, \Gamma_{H}$ respectively, and define $\Delta \Gamma_{d(s)} \equiv \Gamma_{L}-\Gamma_{H}$.

At the present accuracy of measurements, this lifetime difference $\Delta \Gamma_{d}$ can well be ignored. As a result, the measurement and the phenomenology of $\Delta \Gamma_{d}$ have been neglected so far, as compared with the lifetime difference in the $B_{s}$ system for example. However, with the possibility of experiments with high time resolution and high statistics, such as at the LHC, this quantity is becoming more and more relevant.

With the possibility of experiments with high

*Presented by T.Yoshikawa at KEKTC5. time resolution and high statistics, it is worthwhile to have a look at this quantity and make a realistic estimate of the possibility of its measurement (see also [4, 5]).

In [4] we estimated $\Delta \Gamma_{d} / \Gamma_{d}$ including $1 / m_{b}$ contributions and part of the next-to-leading order QCD corrections. We find that adding the latter corrections decreases the value of $\Delta \Gamma_{d} / \Gamma_{d}$ computed at the leading order by a factor of almost 2 . The final result is

$\Delta \Gamma_{d} / \Gamma_{d}=\left(2.6_{-1.6}^{+1.2}\right) \times 10^{-3}$.

The two mass eigenstates of the neutral $B_{d}$ system have slightly different lifetimes. Using another expansion of the partial NLO QCD corrections proposed in [4], we get

$\Delta \Gamma_{d} / \Gamma_{d}=\left(3.0_{-1.4}^{+0.9}\right) \times 10^{-3}$,

where we have used the preliminary values for the bag factors from the JLQCD collaboration [8]. In the error estimation, the errors are the uncertainties on the values of the CKM parameters, of the bag parameters, of the mass of the $b$ quark, and of the measured value of $x_{d}$. Further sources of error are the assumption of naive factorization made for the $1 / m_{b}$ matrix elements, the scale depen- 
dence and the missing terms in the NLO contribution. Although the latter error is decreased in the second estimate by smallness of CKM factors, a complete NLO calculation is definitely desirable for the result to be more reliable.

The most obvious way of trying to measure the width difference is through the semileptonic decays, however we can not extract the quantity which is linear in $\Delta \Gamma_{d} / \Gamma_{d}$. The time measurements of an untagged $B_{d}$ decay to single final state is sensitive only to quadratic terms in $\Delta \Gamma_{d} / \Gamma_{d}$. So this method would involve measuring a quantity as small as $\left(\Delta \Gamma_{d} / \Gamma_{d}\right)^{2} \sim 10^{-5}$, which is too small to measure.

However, combining time measurements from two different final states can enable us to measure quantities linear in $\Delta \Gamma_{d} / \Gamma_{d}$. Indeed, we can measure the ratio of two untagged lifetimes for two final states:

$\frac{\tau_{b_{1}}}{\tau_{b_{2}}}=1+\frac{b_{2}-b_{1}}{2} \frac{\Delta \Gamma_{d}}{\Gamma_{d}}+\mathcal{O}\left[\left(\Delta \Gamma_{d} / \Gamma_{d}\right)^{2}\right]$,

where the $b$ is the quantity depend on the final state. This indicates the necessity of at least two different final states to extract $\Delta \Gamma_{d} / \Gamma_{d}$.

A viable option, perhaps the most efficient among the ones considered in [4], is to compare the measurements of the untagged lifetimes of the semileptonic decay mode $\tau_{S L}$ and of the CPspecific decay modes $\tau_{C P_{ \pm}}$. For each final states, $b_{S L}=0$ and $b_{C P \pm}= \pm \cos \left(2 \phi_{1}\right)$. The ratio between the two lifetimes $\tau_{C P \pm}$ and $\tau_{S L}$ is

$\frac{\tau_{S L}}{\tau_{C P \pm}}=1 \pm \frac{\cos \left(2 \phi_{1}\right)}{2} \frac{\Delta \Gamma_{d}}{\Gamma_{d}}+\mathcal{O}\left[\left(\Delta \Gamma_{d} / \Gamma_{d}\right)^{2}\right]$

The measurement of these two lifetimes should be able to give us a value of $\left|\Delta \Gamma_{d}\right|$, since $\left|\cos \left(2 \phi_{1}\right)\right|$ will already be known to a good accuracy by that time.

Since the CP-specific decay modes of $B_{d}$ (e.g. $J / \psi K_{S(L)}, D^{+} D^{-}$) have smaller branching ratios than the semileptonic modes, and the semileptonic data sample may be enhanced by including the self-tagging decay modes (e.g. $D_{s}^{(*)+} D^{(*)-}$ ) which also have large branching ratios, we expect that the most useful combination will be the measurement of $\tau_{S L}$ through all self-tagging decays and that of $\tau_{C P_{+}}$through the decay $B_{d} \rightarrow$
$J / \psi K_{S}$. After 5 years of LHC running, we should have about $5 \times 10^{5}$ events of $J / \psi K_{S}$ ([9] table 3 ), whereas the number of semileptonic decays, at $\mathrm{LHCb}$ alone, that will be directly useful in the lifetime measurements is expected to be more than $10^{6}$ per year, even with conservative estimates of efficiencies.

At $\mathrm{LHCb}$, the proper time resolution is expected to be as good as $\Delta \tau \approx 0.03$ ps. This indeed is a very small fraction of the $B_{d}$ lifetime $\left(\tau_{B_{d}} \approx 1.5 \mathrm{ps}[10]\right)$, so the time resolution is not a limiting factor in the accuracy of the measurement, and the statistical error plays the dominant role. Taking into account the estimated number of $B_{d}$ produced the measurement of the lifetime difference does not look too hard at first glance. One may infer that if the number of relevant events with the proper time of decay measured with the precision $\Delta \tau$ is $N$, then the value of $\Delta \Gamma_{d} / \Gamma_{d}$ is measured with an accuracy of $1 / \sqrt{N}$. With a sufficiently large number of events $N$, it should be possible to reach the accuracy of $0.5 \%$ or better.

We also point out the interlinked nature of the accurate measurements of $\phi_{1}$ and $\Delta \Gamma_{d} / \Gamma_{d}$ through the conventional gold-plated decay [4.6]. In the future experiments that aim to measure $\phi_{1}$ to an accuracy of 0.005 or better, the corrections due to $\Delta \Gamma_{d}$ will form a major part of the systematic error, which can be taken care of by a simultaneous fit to $\sin \left(2 \phi_{1}\right), \Delta \Gamma_{d}$ and an effective parameter $\bar{\epsilon}$ that comes from the CP violation in $K-\bar{K}$ and $B-\bar{B}$ systems, and also takes care of small theoretical uncertainties.

The calculations of the width difference in $B_{d}$ and in the $B_{s}$ system (as in [i]) run along similar lines. However, there are some subtle differences involved, due to the values of the different CKM elements involved, which have significant consequences. In particular, whereas the upper bound on the value of $\Delta \Gamma_{s}$ (including the effects of new physics) is the value of $\Delta \Gamma_{s}(\mathrm{SM})$ [11], the upper bound on $\Delta \Gamma_{d}$ involves a multiplicative factor in addition to $\Delta \Gamma_{d}(\mathrm{SM})$. Using the definitions $\Theta_{q} \equiv \operatorname{Arg}\left(\Gamma_{21}\right)_{q}, \Phi_{q} \equiv \operatorname{Arg}\left(M_{21}\right)_{q}$, where $q \in\{d, s\}$, we can write

$\Delta \Gamma_{q}=-2\left|\Gamma_{21}\right|_{q} \cos \left(\Theta_{q}-\Phi_{q}\right)$. 
Since the contribution to $\Gamma_{21}$ comes only from tree diagrams, we expect the effect of new physics on this quantity to be very small. We therefore take $\left|\Gamma_{21}\right|_{q}$ and $\Theta_{q}$ to be unaffected by new physics. On the other hand, the mixing phase $\Phi_{q}$ appears from loop diagrams and can therefore be very sensitive to new physics. The effect of new physics on $\Delta \Gamma_{s}$ can be bounded by giving an upper bound on $\Delta \Gamma_{s}$ :

$\Delta \Gamma_{s} \leq \frac{\Delta \Gamma_{s}(\mathrm{SM})}{\cos (2 \Delta \gamma)} \approx \Delta \Gamma_{s}(\mathrm{SM})$

with $2 \Delta \gamma=-\operatorname{Arg}\left[\left(V_{c b}^{*} V_{c s}\right)^{2} /\left(V_{t b}^{*} V_{t s}\right)^{2}\right] \approx-0.03$. Thus, the value of $\Delta \Gamma_{s}$ can only decrease in the presence of new physics 11.

In the $B_{d}$ system, an upper bound for $\Delta \Gamma_{d}$, based on the additional assumption of threegeneration unitarity, can be derived:

$\Delta \Gamma_{d} \leq \frac{\Delta \Gamma_{d}(\mathrm{SM})}{\cos [\operatorname{Arg}(1+\delta f)]}$.

We can calculate the bound (7) in terms of the extent of the higher order NLO corrections. In [we got $|\operatorname{Arg}(1+\delta f)|<0.6$, so that we have the bound $\Delta \Gamma_{d}<1.2 \Delta \Gamma_{d}(\mathrm{SM})$. A complete NLO calculation will be able to give a stronger bound.

We have seen that the ratio of two effective lifetimes can enable us to measure the quantity $\Delta \Gamma_{o b s(d)} \equiv \cos \left(2 \phi_{1}\right) \Delta \Gamma_{d} / \Gamma_{d}$. In the presence of new physics, this quantity is in fact (see eq. (5)) $\Delta \Gamma_{\text {obs }(d)}=-2\left(\left|\Gamma_{21}\right|_{d} / \Gamma_{d}\right) \cos \left(\Phi_{d}\right) \cos \left(\Theta_{d}-\Phi_{d}\right)$. In SM, we get

$$
\begin{aligned}
\Delta \Gamma_{\text {obs }(d)}(\mathrm{SM})=2\left(\left|\Gamma_{21}\right|_{d} / \Gamma_{d}\right) \cos \left(2 \phi_{1}\right) \\
\times \cos [\operatorname{Arg}(1+\delta f)]
\end{aligned}
$$

If $|\delta f|<1.0$, we have $\cos [\operatorname{Arg}(1+\delta f)]>0$ (in fact, from the fit in 12] and our error estimates, we have $\cos [\operatorname{Arg}(1+\delta f)]>0.8)$. Then $\Delta \Gamma_{o b s(d)}(\mathrm{SM})$ is predicted to be positive. New physics is not expected to affect $\Theta_{d}$, but it may affect $\Phi_{d}$ in such a way as to make the combination $\cos \left(\Phi_{d}\right) \cos \left(\Theta_{d}-\Phi_{d}\right)$ change sign. A negative sign of $\Delta \Gamma_{o b s(d)}$ would therefore be a clear signal of such new physics.

It is well known, that the $B_{d}-\bar{B}_{d}$ mixing phase $\Phi_{d}$ is efficiently measured through the decay modes $J / \psi K_{s}$ and $J / \psi K_{L}$. If we take the new physics effects into account, the time-dependent asymmetry is $\mathcal{A}_{C P}=-\sin \left(\Delta M_{d} t\right) \sin \left(\Phi_{d}\right)$; in the SM, we have $\Phi_{d}=-2 \phi_{1}$. The measurement of $\sin \left(\Phi_{d}\right)$ still allows for a discrete ambiguity $\Phi_{d} \leftrightarrow \pi-\Phi_{d}$. It is clear that, if $\Theta_{d}$ can be determined independently of the mixing in the $B_{d}$ system, then measuring $\Delta \Gamma_{o b s(d)}$, which is proportional to $\cos \left(\Phi_{d}\right) \cos \left(\Theta_{d}-\Phi_{d}\right)$, resolves the discrete ambiguity in principle. We note that these features are unique to the $B_{d}$ system.

\section{ACKNOWLEDGMENTS}

The work of C.S.K. was supported by Grant No. 2001-042-D00022 of the KRF. The work of T.Y. was supported in part by the US Department of Energy under Grant No.DE-FG02-97ER41036 .

\section{REFERENCES}

1. M. Beneke, G. Buchalla, C. Greub, A. Lenz and U. Nierste, Phys. Lett. B 459 (1999) 631.

2. M. Beneke and A. Lenz, J.Phys.G G27 (2001) 1219 and references therein.

3. D. Becirevic, hep-ph/0110124 and references therein.

4. A.S. Dighe, T. Hurth, C.S. Kim and T. Yoshikawa, hep-ph/0109088, accepted for publication in Nucl.Phys.B.

5. T. Hurth et al., J. Phys. G 27 (2001) 1277.

6. A.S. Dighe, T. Hurth, C.S. Kim and T. Yoshikawa, hep-ph/0112067.

7. Report of Workshop on B Physics at the Tevatron: Run II and Beyond, hep$\mathrm{ph} / 0201701$.

8. S. Hashimoto and N. Yamada [JLQCD collaboration], hep-ph/0104080.

9. P. Ball et al., hep-ph/0003238.

10. Particle Data Group, D.E. Groom et al., Eur. Phys. J. C15 (2000) 1.

11. Y. Grossman, Phys. Lett. B 380 (1996) 99.

12. S. Mele, hep-ph/0103040. 